\title{
Single-step doxorubicin-selected cancer cells overexpress the ABCG2 drug transporter through epigenetic changes
}

\author{
AM Calcagno', JM Fostel ${ }^{2}, K K W$ To $^{3}$, CD Salcido ${ }^{4}$, SE Martin ${ }^{5}$, KJ Chewning', C-P Wu', L Varticovski ${ }^{4}$, \\ SE Bates ${ }^{3}$, NJ Caplen ${ }^{5}$ and SV Ambudkar*,
}

'Laboratory of Cell Biology, Center for Cancer Research, National Cancer Institute, NIH, DHHS, Bethesda, MD 20892, USA; ${ }^{2}$ Alpha-Gamma Technologies Inc., Raleigh, NC 27609, USA; ${ }^{3}$ Medical Oncology Branch, Center for Cancer Research, National Cancer Institute, NIH, DHHS, Bethesda, MD 20892, USA; ${ }^{4}$ Laboratory of Human Carcinogenesis, Center for Cancer Research, National Cancer Institute, NIH, DHHS, Bethesda, MD 20892, USA; ${ }^{5}$ Gene Silencing Section, Genetics Branch, Center for Cancer Research, National Cancer Institute, NIH, DHHS, Bethesda, MD 20892, USA

\begin{abstract}
Understanding the mechanisms of multidrug resistance (MDR) could improve clinical drug efficacy. Multidrug resistance is associated with ATP binding cassette $(A B C)$ transporters, but the factors that regulate their expression at clinically relevant drug concentrations are poorly understood. We report that a single-step selection with low doses of anti-cancer agents, similar to concentrations reported in vivo, induces MDR that is mediated exclusively by ABCG2. We selected breast, ovarian and colon cancer cells (MCF-7, IGROV-I and S-I) after exposure to 14 or 21 nM doxorubicin for only 10 days. We found that these cells overexpress ABCG2 at the mRNA and protein levels. RNA interference analysis confirmed that ABCG2 confers drug resistance. Furthermore, $A B C G 2$ upregulation was facilitated by histone hyperacetylation due to weaker histone deacetylase I-promoter association, indicating that these epigenetic changes elicit changes in ABCG2 gene expression. These studies indicate that the MDR phenotype arises following low-dose, single-step exposure to doxorubicin, and further suggest that ABCG2 may mediate early stages of MDR development. This is the first report to our knowledge of single-step, low-dose selection leading to overexpression of ABCG2 by epigenetic changes in multiple cancer cell lines.

British Journal of Cancer (2008) 98, 1515 - 1524. doi:10.1038/sj.bjc.6604334 www.bjcancer.com
\end{abstract}

Published online I April 2008

(c) 2008 Cancer Research UK

Keywords: multidrug resistance (MDR); doxorubicin; ABCG2; epigenetics; single-step selection

Cancer remains a major health issue and is responsible for one in four deaths in the United States (Zhou et al, 2001; Jemal et al, 2006). The development of multidrug resistance (MDR) to chemotherapeutic agents plays a major role in the failure of cancer therapy. Resistance to therapy can result from decreased drug uptake, increased DNA repair or drug inactivation (Gottesman et al, 2002). However, the most common mechanism is the overexpression of ATP binding cassette $(\mathrm{ABC})$ drug transporters that protect cells by increasing drug efflux. Several investigators have reported increased $A B C$ transporter expression following drug treatment (reviewed in Lage, 2003). However, a debate surrounds the relevance of multistep and continuous selections with clinically unattainable concentrations commonly used to study cancer drug resistance.

ATP binding cassette transporters are a large superfamily of proteins expressed in normal cells in varying amounts. Some members of this superfamily function to eliminate endogenous and xenobiotic metabolites, and over $12 \mathrm{ABC}$ transporters have been linked to MDR. Importantly, there is a substantial overlap in the list of substrates for $\mathrm{ABCB} 1, \mathrm{ABCG} 2$ and the various $\mathrm{ABCC}$ family members linked with MDR, in spite of significant differences in the primary

*Correspondence: Dr SV Ambudkar;

E-mail: ambudkar@helix.nih.gov

Revised 21 February 2008; accepted 4 March 2008; published online I April 2008 sequences of these transporters (Haimeur et al, 2004). This phenomenon makes treatment of multidrug-resistant cancer unsuccessful despite the various chemotherapeutic drugs that are available.

Cell-based studies of MDR to date have involved establishing multidrug-resistant cancer cell lines through continual or multistep drug selection and using high drug concentrations. Although these cell lines provided an important tool for understanding $\mathrm{ABC}$ transporter function, these selection regimens do not mimic in vivo drug concentrations or dosing frequencies that occur in cancer patients. In this study, we used short, low-dose drug selection of cancer cell lines to more closely simulate in vivo drug concentrations such as those used in the liposomal doxorubicin formulation, which is commonly used as a single-agent therapy for metastatic breast cancer patients with greater cardiac risks (Lorusso et al, 2007) and metastatic ovarian cancer refractory to paclitaxel- and platinum-based chemotherapy (Thigpen et al, 2005). Liposomal doxorubicin is known to have a slow and sustained release of drug from the liposomal carrier with a half-life of $55 \mathrm{~h}$ (Allen et al, 2006). Our in vitro studies utilised doses significantly below the $\mathrm{IC}_{50}$ of doxorubicin for MCF-7 cells; concentrations were kept constant over a 10-day period to mimic the release characteristics from a liposomal carrier. In contrast to multistep exposure to doxorubicin, where either $\mathrm{ABCB} 1$ or $\mathrm{ABCC} 1$ is the dominant $\mathrm{ABC}$ transporter causing MDR (Shen et al, 1986; Barrand et al, 1994; Mehta, 1994), we show here that following single-step selection other transporters, including ABCC4 and $A B C G 2$, are overexpressed. Importantly, ABCG2 was found to 
confer resistance to doxorubicin in breast, ovarian and colon cancer cell lines. Sequencing of ABCG2 in the single-step-selected MCF-7 sublines revealed that our in vitro selection resulted in overexpression of the wild-type ABCG2 and not the gain-offunction mutations either $\mathrm{G}$ or $\mathrm{T}$ at amino acid 482 . While other investigators have reported higher resistance to anthracycline with the 482 mutants (Honjo et al, 2001), we also report increased resistance to doxorubicin in the sublines with wild-type ABCG2 compared to the parental MCF-7 cells in our studies. Overexpression of ABCG2 was also observed following single-step lowdose selection with etoposide. This study shows that ABCG2 may play a critical role in the development of drug resistance.

\section{MATERIALS AND METHODS}

\section{Cell culture}

The MCF-7 breast cancer cell line and the multistep doxorubicinselected subline MCF7/ADR were a gift of Dr Kapil Mehta (MD Anderson Cancer Center, Houston, TX, USA) (Mehta, 1994). The multistep doxorubicin-selected MCF-7 cells were cultured in high-dose doxorubicin ( $860 \mathrm{~nm}$ ) every alternate passage as previously described (Mehta, 1994). The generation of MCF-7/ ADR-VP3000 and MCF7/FLV1000 cell lines by multistep selection was described previously (Chen et al, 1990; Robey et al, 2001). Spectral karyotyping of these cells matched those described by others (Kytola et al, 2000). Parental HEK293 cells (293 human embryonic kidney cells) and the MRP4-overexpressing HEK293/ 4.63 cells were gifts of $\mathrm{P}$ Borst (The Netherlands Cancer Institute, Amsterdam, The Netherlands) (Wielinga et al, 2002). HEK293/4.63 cells were reported to express significantly more MRP4 (Reid et al, 2003) without overexpression of other $A B C$ drug transporters and were cultured as previously described (Wu et al, 2005). The ovarian IGROV-1 cells were obtained from the Developmental Therapeutics Program, NCI. S-1 cells, cloned from LS174T colon carcinoma cells, were described previously (Rabindran et al, 1998).

\section{Drug selection protocol}

Drug-resistant MCF-7, IGROV-1 and S-1 clones were established, employing a single-step selection with either 14 or $21 \mathrm{~nm}$ doxorubicin or $300 \mathrm{~nm}$ etoposide treatment for 10 days followed by culturing in drug-free medium. Briefly, 10000 cells were seeded in a $100 \times 20 \mathrm{~mm}$ tissue culture dish with drug. Medium with drug was changed three times during the selection and, subsequently, the cells were maintained in medium without drug. Individual cells were selected randomly using sterile cloning disks pretreated in trypsin and placed in separate wells within a 24 -well plate. Clones were grown to confluency and expanded in drug-free media. Expanded clones were retested for drug resistance before any further studies. Detailed characterisation of all clones began approximately $8-12$ weeks after single-step selection. The resistant phenotype in isolated clones was examined 24 weeks after initial treatment and was found to be stable.

\section{RNA isolation and quantitative RT - PCR}

RNA was isolated from cells grown in six-well plates to characterise $\mathrm{ABC}$ transporter expression in all cell lines as described previously ( $\mathrm{Wu}$ et al, 2005). Real-time quantitative RT - PCR (qRT - PCR) was performed using the LightCycler RNA Master SYBR Green kit and the LightCycler 480 (Roche Biochemicals, Indianapolis, IN, USA). Plasma membrane calcium ATPase 4 (PMCA4) was used as the reference gene (Calcagno et al, 2006). Specific PCR conditions and primer sequences for genes are described in Supplementary Information.

\section{Western blotting}

For western blotting assays, equivalent numbers of cells were harvested and lysed as described previously (Wu et al, 2005). Nitrocellulose membranes were probed with the appropriate primary antibodies specific to the protein of interest. The following antibodies were used: C219 (1:2000), ABCB1 (Kartner et al, 1985); M2 III-6 (1:50) (Alexis Biochemical, Lausen, Switzerland), ABCC2 (Paulusma et al, 1996); anti-BCRP $(1: 1000)$ (Kamiya Biochemical, Seattle, WA, USA), ABCG2 (Maliepaard et al, 2001); and M4 1-80 $(1: 200)$ (Kamiya Biochemical), ABCC4 (Leggas et al, 2004). Glyceraldehyde-3-phosphate dehydrogenase (GAPDH; $1 \mu \mathrm{g} \mathrm{ml}^{-1}$ ) (Zymed Laboratories, San Francisco, CA, USA) was used as a loading control.

\section{Confocal imaging}

MCF-7 parental cells and $21 \mathrm{~nm}$ single-step-selected cells were seeded at 10000 cells per well on Lab-Tek chamber slides with covers (Nunc, Rochester, NY, USA). After $48 \mathrm{~h}$, the chambers were washed twice with PBS (Gibco-Invitrogen, Carlsbad, CA, USA), fixed (IntraPrep kit, Beckman Coulter, Fullerton, CA, USA) for $15 \mathrm{~min}$ at room temperature (RT), washed three times with wash buffer (PBS/0.1\% bovine serum albumin) and blocked for $1 \mathrm{~h}$ at RT with wash buffer. Following two washes with PBS, a $1: 100$ solution of the 5D3 clone of the mouse anti-human ABCG2 antibody (eBioscience, San Diego, CA, USA) in wash buffer was added to all cell chambers for $1 \mathrm{~h}$ at RT followed by three washes with wash buffer. The anti-mouse Alexa Fluor 488-conjugated antibody (Invitrogen, Carlsbad, CA, USA) (diluted 1:250 in wash buffer) was incubated with the cells for $30 \mathrm{~min}$ at RT. Following two washes with wash buffer, the chambers and seal were removed. Slides were air dried and mounted in aqueous mounting media with 4',6-diamidino-2-phenylindole (DAPI) (Vector Laboratories Inc., Burlingame, CA, USA). Images were acquired using an LSM 510 confocal system mounted on a Zeiss Axiovert $100 \mathrm{M}$ microscope with an oil immersion Plan-Apochromat $\times 63 / 1.4$ NA DIC objective lens (Carl Zeiss, Thornwood, NY, USA). Excitation of Alexa Fluor 488 was performed using the $488 \mathrm{~nm}$ line from a $25 \mathrm{~mW}$ argon laser while excitation of DAPI was performed by using the $364 \mathrm{~nm}$ line from an $80 \mathrm{~mW}$ UV laser. The Alexa Fluor 488 emission was collected using a long-pass $505 \mathrm{~nm}$ filter and the DAPI emission was collected using a band pass $390-$ $465 \mathrm{~nm}$ filter.

\section{Cytotoxicity assay}

Sensitivities of cell lines to various chemicals were examined using the Cell-Counting Kit (CCK) technique as detailed previously (Ishiyama et al, 1996). Cells were plated at a density of 1000-5000 cells per well in 96-well plates containing $100 \mu \mathrm{l}$ of culture medium. After $24 \mathrm{~h}$ incubation at $37^{\circ} \mathrm{C}$, drugs were added into wells to a final volume of $200 \mu \mathrm{l}$ per well and incubated for an additional $72 \mathrm{~h}$ CCK reagent was then added into each well and incubated for $4 \mathrm{~h}$ before reading at a wavelength of $450 \mathrm{~nm}$. IC IC $_{50}$ values were calculated from dose-response curves obtained from at least three independent experiments.

\section{siRNA design and silencing of $A B C G 2$ expression}

The siRNAs employed were designed and synthesised by Qiagen Inc. (Germantown, MD, USA). The ABCG2-2 siRNA duplex (siG2-2) consisted of $5^{\prime}$-GGAUAAGCCACUCAUAGAAdtdT (sense) and $5^{\prime}$-UUCUAUGAGUGGCUUAUCCdTdG (antisense) strands. The negative siRNA duplex (siNeg) consisted of $5^{\prime}$-ACGUGACACGU UCGGAGAAdTdT and 5'-UUCUCCGAACGUGUCACGUdTdT strands. For siRNA transfections, siRNA (1.25 or 5 pmol) was added to individual wells of a 96-well plate in $25 \mu \mathrm{l}$ serum-free 
DMEM. Oligofectamine (0.7 $\mu$; Invitrogen) was subsequently added to siRNA-containing wells in $25 \mu \mathrm{l}$ serum-free DMEM to give a final lipid/siRNA ratio of $2: 1(\mathrm{w} / \mathrm{w})$. The resulting mixture was allowed to complex for $30 \mathrm{~min}$ at ambient temperature. Cells (5000 cells per well - RNA analysis; 1000 cells per well cytotoxicity assay) were added in $50 \mu \mathrm{l}$ DMEM supplemented with $20 \%$ FBS to yield transfection mixtures of 12.5 or $50 \mathrm{~nm}$ siRNA in DMEM containing 10\% FBS. This final mixture was incubated at ambient temperature for $45 \mathrm{~min}$ before being placed at $37^{\circ} \mathrm{C}$ in a humidified atmosphere containing $5 \% \mathrm{CO}_{2}$. For initial screening of siRNA and protein analysis, the transfection protocol was performed as described above using six-well plates and the reagents were scaled up 30 -fold using $50 \mathrm{~nm}$ siRNA. ABCG2 mRNA levels were analysed $48 \mathrm{~h}$ after transfection by real-time RT - PCR for the initial siRNA screening. RNA levels for the comparison of 12.5 and $50 \mathrm{~nm}$ were analysed $48 \mathrm{~h}$ after transfection using the QuantiGene Reagent System (Panomics, Fremont, CA, USA) and normalised to cyclophilin B (PPIB) mRNA. The results reflect the average and s.d. of at least five replicate experiments. ABCG2 protein levels were measured by western blotting as described above $48 \mathrm{~h}$ after siRNA transfection. For drug analysis, cells were grown for $48 \mathrm{~h}$ before adding various concentrations of mitoxantrone for $72 \mathrm{~h}$ as described above.

\section{Chromatin immunoprecipitation assay}

Chromatin immunoprecipitation (ChIP) assays were performed on MCF-7, MCF7/FLV1000 and five single-step doxorubicin-selected clones. Briefly, cells $\left(1 \times 10^{6}\right)$ were crosslinked with $1 \%$ formaldehyde for $10 \mathrm{~min}$ at $37^{\circ} \mathrm{C}$, quenched with $0.125 \mathrm{M}$ glycine for $5 \mathrm{~min}$ at $\mathrm{RT}$ and rinsed in ice-cold PBS containing $5 \mathrm{~mm}$ sodium butyrate (Sigma, St Louis, MI, USA). Cells were scraped and re-suspended in a lysis buffer (Active Motif, Carlsbad, CA, USA) with the addition of complete protease inhibitor cocktail (Roche Applied Science, Indianapolis, IN, USA). DNA-protein complexes were sheared using an enzymatic shearing kit (Active Motif) to yield DNA fragments below $800 \mathrm{bp}$, as determined by agarose gel electrophoresis. Chromatin immunoprecipitations were carried out overnight at $4^{\circ} \mathrm{C}$ with $10 \mu \mathrm{g}$ of anti-acetylated $\mathrm{H} 3(\mathrm{AcH} 3), 5 \mu \mathrm{g}$ of anti-histone deacetylase 1 (HDAC1; Upstate Biotechnology, Charlottesville, VA, USA) and $2 \mu \mathrm{g}$ of anti-RNA polymerase II (Pol II; Santa Cruz Biotechnology, Santa Cruz, CA, USA). Primer sets encompassing approximately $1.6 \mathrm{~kb}$ of the $A B C G 2$ promoter were used to map changes in histone acetylation after drug selection. The amount of immunoprecipitated DNA was assessed by quantitative PCR, using primers spanning the proximal ( -293 to $-139 \mathrm{nt})$ and distal $(-1527$ to $-1268 \mathrm{nt})$ promoter region of $A B C G 2$, and compared to the amount of input DNA prior to immunoprecipitation. The numbering of the nucleotides was assigned relative to the transcriptional start site designated in GenBank sequence AF151530. Amplification of immunopreci- pitated DNA was achieved using previously described methods (To et al, 2006).

\section{RESULTS}

\section{Single-step doxorubicin selection results in ABCG2 overexpression}

The gene expression profiles of select $\mathrm{ABC}$ transporters (ABCB1, $\mathrm{ABCC} 1, \mathrm{ABCC} 2, \mathrm{ABCC} 4$ and $\mathrm{ABCG} 2$ ) linked to MDR (PerezTomas, 2006) were assayed using GRT-PCR in MCF-7 cells subjected to drug selection. These transporters have been associated with the MDR phenotype in various cell lines (Perez-Tomas, 2006). Briefly, ten thousand cells (in a $100 \times 20 \mathrm{~mm}$ dish) were treated for 10 days with low-dose doxorubicin. Drug selection with either 14 or $21 \mathrm{~nm}$ doxorubicin was performed. These doses were chosen because they are significantly below the $\mathrm{IC}_{50}$ of doxorubicin for MCF-7 cells, which is $\sim 90 \mathrm{~nm}$ (Table 1). Following the 10-day drug selection, single isolated cells were chosen randomly for expansion. The $\mathrm{ABC}$ transporter expression patterns for six doxorubicin-resistant MCF-7 clones selected with $14 \mathrm{~nm}$ doxorubicin and one $21 \mathrm{~nm}$ doxorubicin-resistant clone are shown in Figure 1A. We also performed selections at higher concentrations of doxorubicin (70, 140 and $210 \mathrm{~nm})$. No MCF-7 cells survived this selection pressure, suggesting that pre-existing cells exhibiting high levels of $\mathrm{ABC}$ transporters within the MCF-7 parental population were not present. In contrast to the previously established multistep-selected line MCF7/ADR (Mehta, 1994), most of the low-concentration clones did not express $A B C B 1$ (Figure 1A). Only the $14 \mathrm{nM}$ doxorubicin-selected MCF-7 clone 13 showed a slight increase in $\mathrm{ABCB} 1$ at the mRNA level; yet, no detectable levels of $\mathrm{ABCB} 1$ protein were found in this clone (Figure 2A). On the contrary, all single-step-selected clones showed overexpression of $A B C G 2$ (4- to 30-fold) when compared to their parental MCF-7 cells.

To determine if this pattern of expression of MDR-associated $\mathrm{ABC}$ transporters following below $\mathrm{IC}_{50}$ selection was drug and cell line independent, we selected MCF-7 cells with a single-step selection using $300 \mathrm{~nm}$ etoposide and two additional cancer lines, IGROV-1 ovarian cancer cells and S-1 colon tumor cells, with 14 and $21 \mathrm{~nm}$ doxorubicin, respectively (Figure 1B-D). With lower etoposide concentrations of 50, 100 and $200 \mathrm{nM}$, all MCF-7 parental cells were able to survive the selection pressure. For IGROV-1 cells, no parental cells survived the $21 \mathrm{~nm}$ selection. We also studied five sublines derived from IGROV-1 cells obtained using $14 \mathrm{~nm}$ doxorubicin as well as S-1-resistant clones, obtained at a $21 \mathrm{~nm}$ concentration of doxorubicin. In all cases, ABCG2 was the dominant overexpressed gene; the etoposide-selected MCF-7 cells and the doxorubicin-selected S-1 increased $A B C G 2$ mRNA levels 43 -fold while IGROV-1 cells exhibited a nearly 400 -fold increase. This suggests that $A B C G 2$ overexpression is an early molecular

Table I Sensitivity of clones to doxorubicin and mitoxantrone

\begin{tabular}{|c|c|c|c|c|c|c|c|c|}
\hline & $\begin{array}{c}\text { Parental } \\
\text { MCF-7 }\end{array}$ & I4 nM clone I & I 4 nM clone 2 & 14 nM clone 6 & I4 nM clone II & I 4 nM clone I 3 & $14 \mathrm{~nm}$ clone 16 & $21 \mathrm{~nm}$ clone I \\
\hline \multicolumn{9}{|l|}{ Doxorubicin } \\
\hline $\begin{array}{l}\text { IC } C_{50}(\mathrm{nM}) \\
\text { s.d. }\end{array}$ & $\begin{array}{l}89.7 \\
28.2\end{array}$ & $\begin{array}{r}111.1 \\
27.3\end{array}$ & $\begin{array}{r}118.9 \\
50.8\end{array}$ & $\begin{array}{r}173.7 \\
46.3\end{array}$ & $\begin{array}{r}284.7 \\
56.7\end{array}$ & $\begin{array}{r}264.4 \\
65.6\end{array}$ & $\begin{array}{r}260.7 \\
51.8\end{array}$ & $\begin{array}{r}323.0 \\
63.4\end{array}$ \\
\hline \multicolumn{9}{|l|}{ Mitoxantrone } \\
\hline $\begin{array}{l}\text { IC } C_{50}(\mathrm{nM}) \\
\text { s.d. }\end{array}$ & $\begin{array}{r}15.6 \\
6.0\end{array}$ & $\begin{array}{r}33.1 \\
6.1\end{array}$ & $\begin{array}{r}15.4 \\
7.5\end{array}$ & $\begin{array}{l}319.1 \\
114.8\end{array}$ & $\begin{array}{r}158.6 \\
11.4\end{array}$ & $\begin{array}{r}220.1 \\
56.2\end{array}$ & $\begin{array}{r}172.4 \\
40.3\end{array}$ & $\begin{array}{r}1407.0 \\
310.2\end{array}$ \\
\hline
\end{tabular}

$I C_{50}$ values are given in $\mathrm{nM}$ and are mean \pm s.d. The $I \mathrm{C}_{50}$ values were calculated from dose-response curves obtained from at least three independent experiments. $C$ ytotoxicity assays were performed as described in Materials and Methods. 

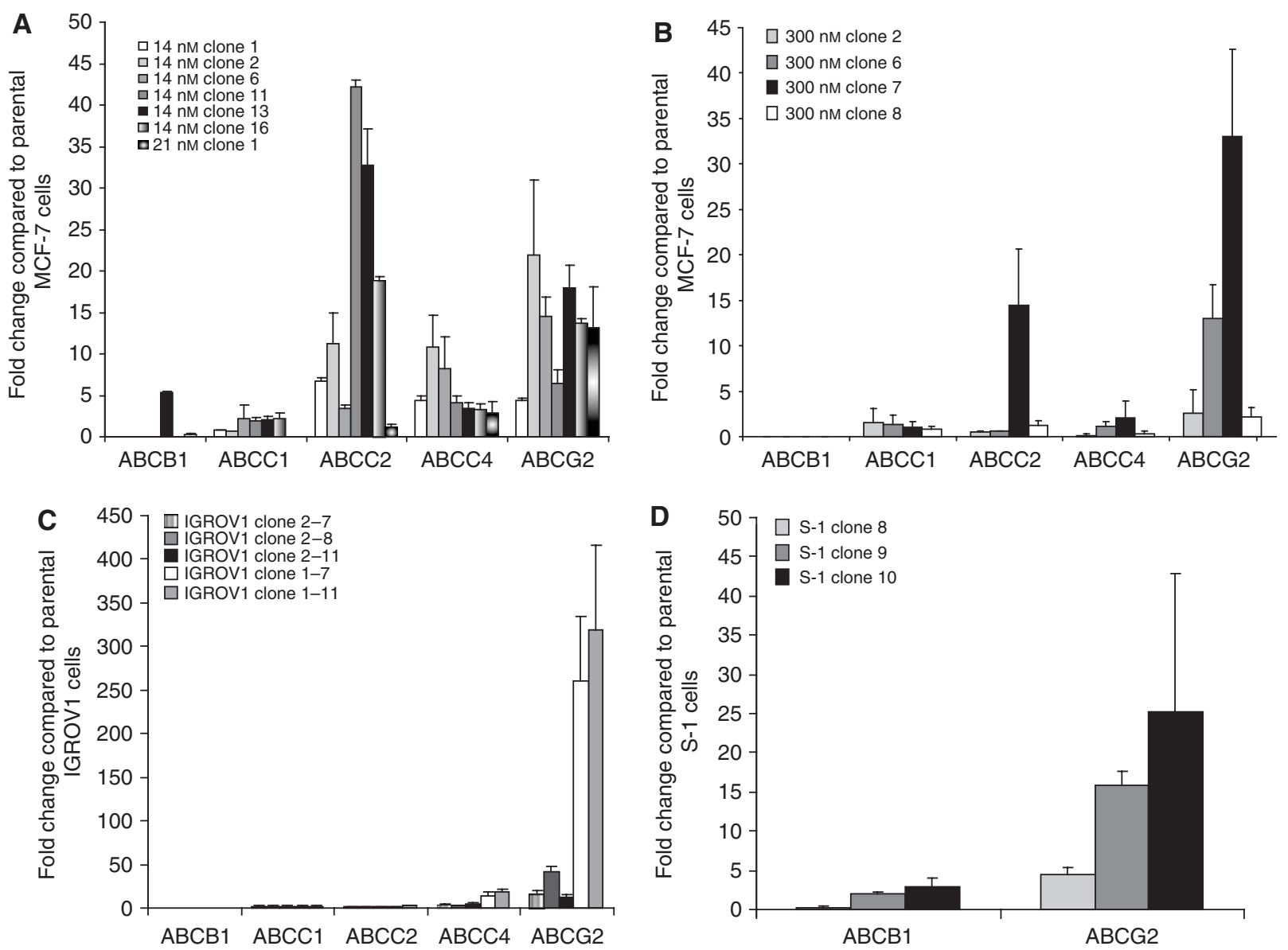

Figure I Single-step drug-selected clones overexpress ABCG2 mRNA. (A) Characterisation of selected ABC transporter gene expression levels in several single-step doxorubicin-resistant MCF-7 clones, described in Materials and Methods. The average fold change compared to parental MCF-7 cells \pm s.d. $(n=4)$ was calculated using delta delta $C_{t}$ method from real-time RT-PCR data. The colour-coded key for the clones is given in the figure. (B) Single-step etoposide-resistant MCF-7 clones are described in Materials and Methods. The average fold change compared to parental MCF-7 cells \pm s.d. ( $n=4)$ was calculated using delta delta $C_{t}$ method from real-time RT-PCR data. The colour-coded key for the clones is given in the figure. (C) Doxorubicin-resistant IGROV-I clones were established employing a single-step selection with $14 \mathrm{nM}$ doxorubicin treatment as described previously. The average fold change compared to parental IGROV-I cells \pm s.d. $(n=4)$ was calculated using delta delta $C_{t}$ method from real-time RT-PCR data. The colour-coded key for the clones is given in the figure. (D) Doxorubicin-resistant S-I clones were established employing a single-step selection with 21 nM doxorubicin. The average fold change compared to parental S-I cells \pm s.d. $(n=4)$ was calculated using delta delta $C_{t}$ method from real-time RT-PCR data. The key for the clones is given in each panel.

marker of the development of MDR in these three cancer cell types. Two other $\mathrm{ABC}$ transporters $A B C C 2$ and $A B C C 4$ were also overexpressed in some sublines of MCF-7 cells selected with doxorubicin, exhibiting a 2- to 24-fold and 3- to 10-fold overexpression of mRNA when compared to the parental MCF-7 cells, respectively.

To examine if the protein expression of these transporters was also changed, several immunoblots were performed. Consistent with the mRNA analysis, neither the parental cell line nor any of the single-step doxorubicin-resistant MCF-7 clones including clone 13, which showed a slight increase in ABCB1 mRNA, expressed $\mathrm{ABCB} 1$ protein at detectable levels whereas the MCF7/ ADR cells expressed high levels of ABCB1 (Figure 2A, top panel). Surprisingly, no ABCC2 protein was detected in any of the MCF-7 sublines, although several clones showed a 2- to 24-fold increase of mRNA compared to the parental MCF-7 cells (Figure 2A, second panel). The reason for the lack of correlation is not yet clear. $\mathrm{ABCC} 4$ protein was detected in all clones examined (Figure 2A, third panel). Five of these doxorubicin-selected MCF-7 clones showed enhanced levels of ABCG2 protein compared to parental MCF-7 cells, with two clones, $14 \mathrm{~nm}$ clone 11 and $21 \mathrm{~nm}$ clone, showing very high levels (Figure 2A, bottom panel). This analysis showed enhanced mRNA and protein levels, which was consistent with the increase in the function and cell surface expression of ABCG2 in these MCF-7 single-step clones. Interestingly, overexpression of ABCG2 was retained in the absence of long-term selection, as both ABCG2 mRNA and protein levels were found to be increased in cells grown for 24 weeks in the absence of doxorubicin following the initial drug treatment (data not shown).

To further support these western blotting results, which showed that the ABCG2 protein was found in the single-step clones, confocal microscopy studies were also performed to determine if the ABCG2 protein in the single-step-selected cells was properly localised to the plasma membrane. Using the 5D3 antibody, which detects an extracellular epitope of ABCG2 (Zhou et al, 2001), the cell surface expression of ABCG2 was detected in non-permeabilised cells. The MCF-7 parental cells did not show binding of 5D3 (Figure 2B, left panel); however, the $21 \mathrm{~nm}$ clone showed a distinct pattern of binding of 5D3 (Figure 2B, right panel) in intact cells. This suggested that ABCG2 predominately localised to the plasma membrane of these cells, where it can be functional as an efflux transporter. 
A

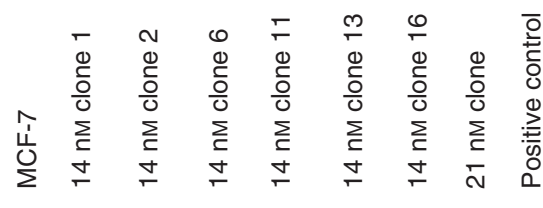

$\mathrm{ABCB} 1$

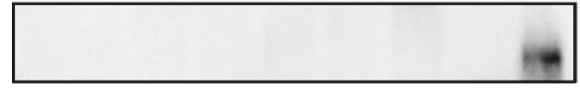

ABCC2

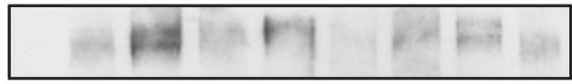

ABCC4

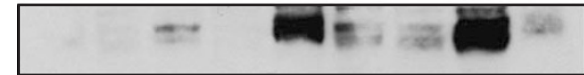

ABCG2

B
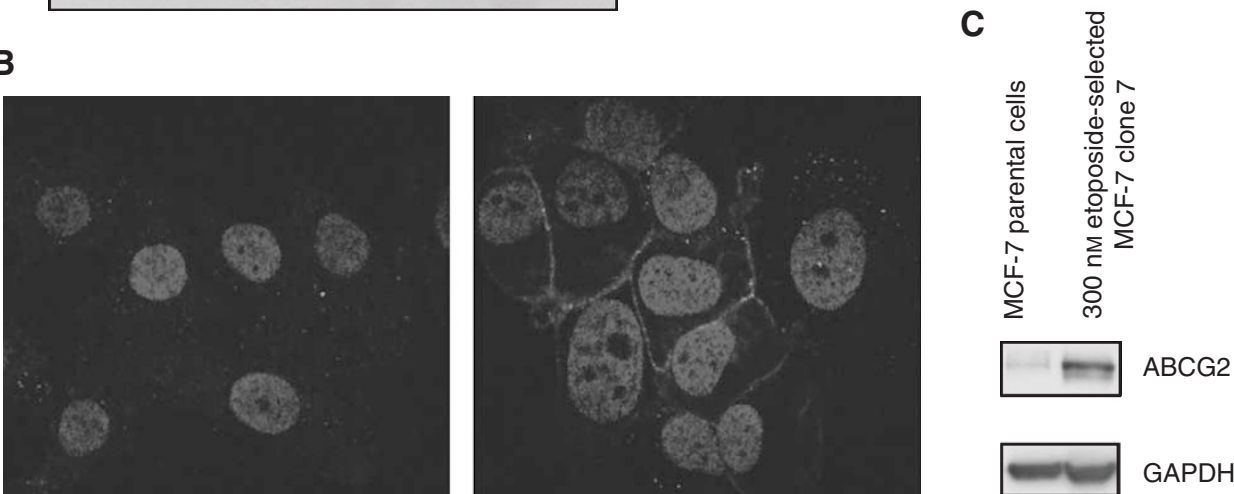

Figure 2 Single-step drug-selected MCF-7 clones overexpress ABCG2 protein. (A) Western blotting analysis of selected ABC drug transporters in doxorubicin-selected MCF-7 clones. Lanes I -8 are the same in all panels and only lane 9 varies in each panel. A total of I 00000 cells were loaded per lane for lanes I -8. Lane I, parental MCF-7; lane 2, I 4 nM clone I; lane 3, I 4 nM clone 2; lane 4, I 4 nM clone 6; lane 5, I 4 nM clone II; lane 6, I 4 nM clone I3; lane 7, I 4 nM clone 16; and lane 8, 21 nM clone. Top panel lane 9, 10000 MCF7/ADR cells (positive control); second panel lane 9, 20000 LLCPK-ABCC2 cells (positive control); third panel lane 9, 2000 ABCC4-HEK293 cells (positive control); and bottom panel lane 9, 5000 MCF7/FLVI 000 cells (positive control). Immunoblotting with antibodies specific for each transporter (as indicated on the right side of each panel) was carried out as described in Materials and Methods. (B) Cell surface localisation of ABCG2 in 21 nM doxorubicin-selected MCF-7 clone. 5D3 binding in MCF-7 (left panel) and 21 nM clone cells (right panel) is shown. Confocal imaging was performed on permeabilised cells following binding with the ABCG2-specific 5D3 antibody (Zhou et al, 200I) as described in Materials and Methods. Nuclei were stained with DAPI, blue stain (showing as light grey in print); Alexa-Fluor 488 secondary antibody (green) (white in print) was used to localise 5D3 binding. (C) Western blotting analysis of ABCG2. A total of I00 000 cells were loaded per lane for lanes I and 2. Lane I, MCF-7; lane 2, 300 nM etoposide-selected MCF-7 clone 7. Glyceraldehyde-3-phosphate dehydrogenase was used as a loading control.

To confirm that the single-step clones of etoposide-selected MCF-7 cells, doxorubicin-selected IGROV-1 and doxorubicinselected S-1 cells also showed an overexpression of ABCG2 at the protein level, western blots were performed on these sublines. The ABCG2 protein expression of the etoposide-selected MCF-7 clone 7 following normalisation to GAPDH levels was 1.9-fold greater than parental MCF-7 cells (Figure 2C). In addition, the IGROV-1 parental cells show no detectable expression of ABCG2 while clones $1-7$ and 1-11, the two clones showing the greatest increase in $A B C G 2$ mRNA expression, showed ABCG2 protein expression (data not shown).

Overexpression of ABCG2 in vitro can cause a mutation at position $482(\mathrm{R} 482 \rightarrow \mathrm{G}$ or $\mathrm{T})$, which alters the substrate specificity of this transporter (Honjo et al, 2001; Allen et al, 2002). To determine if this mutation was also present following low-dose, single-step selection, ABCG2 was sequenced following PCR with primers covering the region (aa 438-561) (see the Materials and Methods section in Supplementary Information). Several clones and MCF-7/ADR-VP3000 cells were sequenced. Sequence data showed that only the wild-type $A B C G 2$ was present in all clones (data not shown), while the MCF-7/ADR-VP3000 cells had the mutation at position $\mathrm{R} 482 \rightarrow \mathrm{T}$ (data not shown), consistent with what has been reported previously (Honjo et al, 2001).
To show the functional activity of the single-step doxorubicinselected clones, we evaluated drug resistance. We examined the cytotoxicity of doxorubicin for the parental MCF-7 cells and the single-step-selected $14 \mathrm{~nm}$ clones and the $21 \mathrm{~nm}$ clone. The $\mathrm{IC}_{50}$ value for the parental cells was approximately $90 \mathrm{~nm}$, while for the $14 \mathrm{~nm}$ doxorubicin-selected clones 1 and 2 , the $\mathrm{IC}_{50}$ values were only 1.2 -fold higher, indicating no increase in resistance to doxorubicin (Table 1). In contrast, the remaining $14 \mathrm{nM}$ doxorubicin-selected MCF-7 clones demonstrated a 2- to 3.2-fold increase in resistance to doxorubicin. The $21 \mathrm{~nm}$ doxorubicinselected clone was nearly four-fold more resistant to doxorubicin and over 90 -fold more resistant to mitoxantrone (Table 1). Our data agree with previous reports that demonstrate more resistance towards mitoxantrone than doxorubicin in cell lines expressing the wild-type ABCG2 (Honjo et al, 2001). Thus, these clones show not only resistance to doxorubicin but also demonstrate MDR.

\section{ABCC4 does not confer resistance to doxorubicin}

To determine if the expression of $A B C C 4$ can confer resistance to either doxorubicin or mitoxantrone, additional studies using ABCC4-overexpressing HEK293 cells were performed. We previously demonstrated that only $\mathrm{ABCC} 4$ is overexpressed in these 

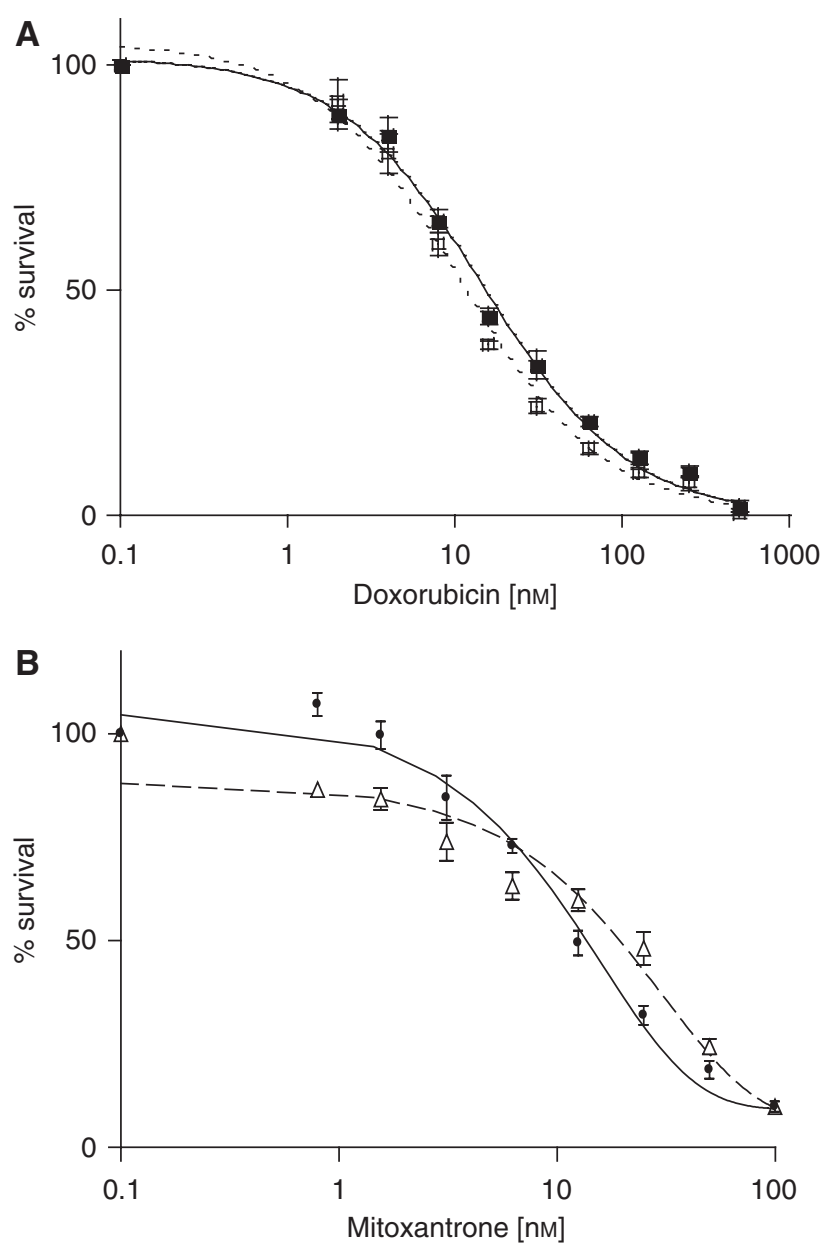

Figure 3 ABCC4 does not confer resistance to doxorubicin or mitoxantrone. (A) Cytotoxicity assays for ABCC4-overexpressing HEK293 (ם) and parental HEK293 ( $\square$ ) cells with doxorubicin. Doseresponse curves were derived from three independent experiments using the CCK-8 assay. (B) Cytotoxicity assays for ABCC4-overexpressing

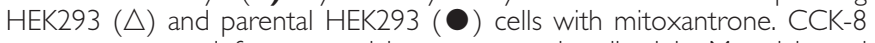
reagent was used for cytotoxicity assays as described in Materials and methods. Dose-response curves were derived from three independent experiments. In both $(\mathbf{A})$ and $(\mathbf{B})$, error bars indicate s.d. $(n=3)$.

cells (Wu et al, 2005). Cytotoxicity assays with doxorubicin were performed comparing HEK293 cells to ABCC4-overexpressing HEK293 cells, and no significant differences were found in the ABCC4-overexpressing HEK293 compared to the parental HEK293 cells, $\mathrm{IC}_{50}$ values $14.9 \pm 1.2$ and $10.7 \pm 1.0 \mathrm{~nm}$, respectively (Figure 3A). Additional cytotoxicity assays were performed to determine if ABCC4 can confer resistance to mitoxantrone (Figure 3B). Similar to the assays with doxorubicin, no significant differences were found in the ABCC4-overexpressing HEK293 compared to the parental HEK293 cells. This suggests that ABCC4 is not responsible for the observed resistance to doxorubicin or mitoxantrone in single-step-selected clones.

\section{ABCG2 confers drug resistance to single-step doxorubicin- selected MCF-7 cells}

To further evaluate if ABCG2 was conferring resistance to doxorubicin in the $21 \mathrm{nM}$ MCF-7 clone, we performed cytotoxicity assays in the presence and absence of fumitremorgin C (FTC) (Rabindran et al, 2000), a specific inhibitor of ABCG2. With the addition of $5 \mu \mathrm{M}$ FTC, we were able to reverse the ABCG2-mediated resistance to doxorubicin (Figure 4A) to levels comparable to those of the parental MCF-7 cells (Table 1). The addition of FTC resulted in a 2.1-fold enhancement of the toxicity of doxorubicin on the $21 \mathrm{~nm}$ doxorubicin-selected MCF-7 cells. This indicates that the inhibition of ABCG2 results in greater toxicity of doxorubicin in these cells.

To confirm that $\mathrm{ABCG} 2$ was the $\mathrm{ABC}$ transporter conferring resistance to the single-step clones, RNA interference (RNAi) studies were also performed. Standard characterisation analysis was conducted to determine the degree of silencing mediated by a synthetic siRNA corresponding to ABCG2 (siG2-2). Using $50 \mathrm{~nm}$ siRNA, ABCG2 mRNA was decreased by nearly 40 -fold compared to a negative siRNA in the $21 \mathrm{~nm}$ MCF7 clone and ABCG2 protein expression was almost completely suppressed (Figure 4B). A lower concentration of siRNA was also examined, and comparable silencing was also observed at an mRNA level using $12.5 \mathrm{~nm}$ siRNA (Figure 4C).

Following validation, cytotoxicity assays were performed on cells treated with siG2-2 or negative control siRNA. The siG2-2 siRNA induced a shift of the drug response curve to the left, indicating less resistance to mitoxantrone (Figure $4 \mathrm{D}$ ). The $\mathrm{IC}_{50}$ value for the siG2-2-treated cells was three-fold less than that for the negative siRNA-treated cells. Silencing with siG2-2 restored sensitivity to approximately $80-85 \%$ of the parental levels. Together with the results from the FTC cytotoxicity studies, this suggests that ABCG2 is critical for the drug resistance in the singlestep $21 \mathrm{~nm}$ doxorubicin-selected MCF-7 cells.

Histone acetylation is modified at the ABCG2 locus in the single-step doxorubicin-selected clones

To determine whether expression of ABCG2 was associated with epigenetic changes such as histone acetylation, we performed ChIP assays to measure the interaction of the protein with a specific DNA sequence in vivo (Kuo and Allis, 1999). We used ChIP to investigate the level of AcH3, HDAC1 and RNA Pol II associated with different segments of the $A B C G 2$ gene. Compared with the parental MCF-7 cells, the enrichment of $\mathrm{H} 3$ acetylation to the proximal $A B C G 2$ promoter was enhanced more than 10 -fold but there was less association of HDAC1 (approximately 50\% decrease) in the selected clones and in a well-characterised resistant subline MCF7/FLV1000 (Robey et al, 2001) (Figure 5A and $\mathrm{B}$ ). The loss of HDAC1 and the stronger binding of Pol II to the proximal promoter correlated well with the enhanced transcription of $A B C G 2$ in these cells. As a control, the weak association of Pol II, AcH3 and HDAC1 to the distal ABCG2 promoter remained similar in the parental, resistant MCF7/ FLV1000 and the doxorubicin-selected clones. The GAPDH promoter was employed as an additional control. Compared with the parental MCF-7 cells, the drug-selected clones expressed a similar level of GAPDH, as determined by RT-PCR (data not shown). To determine whether drug selection-related histone $\mathrm{H} 3$ hyperacetylation was specific to the $A B C G 2$ promoter, we assessed the association of $\mathrm{Pol} \mathrm{II}, \mathrm{AcH} 3$ and $\mathrm{HDAC1}$ with the housekeeping gene GAPDH promoter. Accordingly, ChIP analysis did not reveal any appreciable difference in the binding of Pol II, AcH3 and HDAC1 to the GAPDH promoter between the parental and the drug-selected sublines (Figure 5A and B). This correlated with the strong binding of Pol II and $\mathrm{AcH} 3$ and the weak association of HDAC1 to the proximal promoter of GAPDH that did not change from the parental to the selected clones. These results demonstrate that increased histone $\mathrm{H} 3$ acetylation in the proximal $A B C G 2$ promoter in response to doxorubicin selection was gene-specific.

In contrast, immunoblot analysis of whole-cell lysates demonstrated that drug selection did not globally change the level of histone $\mathrm{H} 3$ acetylation in the doxorubicin-selected clones or in MCF7/FLV1000 (Figure 5C). The lack of global changes in histone acetylation supports the notion that regional accumulation of 

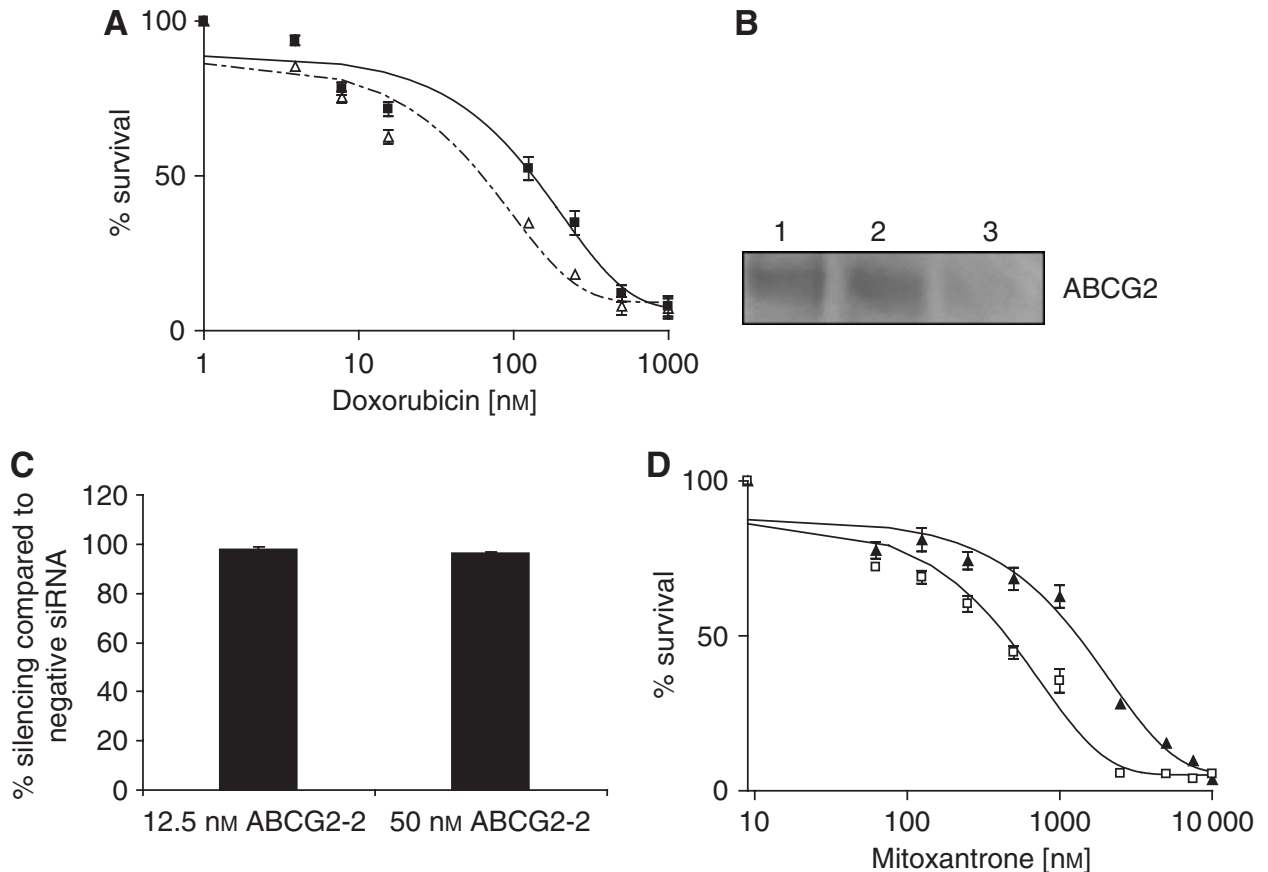

Figure 4 ABCG2 confers resistance to 21 nM single-step doxorubicin-selected clone. (A) Cytotoxicity assays using doxorubicin to evaluate the effect of inhibiting ABCG2 in the $21 \mathrm{nM}$ single-step clone with $5 \mu \mathrm{M}$ FTC. Dose-response curves were derived from six independent experiments using the CCK-8 assay for $2 \mathrm{I}$ nM cells with $5 \mu \mathrm{M} F \mathrm{FT}(\triangle)$ and without $5 \mu \mathrm{M} \mathrm{FTC}(\boldsymbol{\square})$. The mean values from six independent experiments are shown with error bars as s.e.m. (B) Western blotting analysis of ABCG2 protein using BXP-2I antibody following no treatment (lane I), 50 nM negative siRNA treatment (lane 2) and 50 nM G2-2 siRNA treatment (lane 3). (C) Examination of two concentrations of siG2-2 siRNA on silencing of ABCG2. Levels of ABCG2 following siRNA treatment were analysed using the QuantiGene Reagent System (Panomics). Levels were normalised to cyclophilin B (PPIB) mRNA and results reflect the average and s.d. $(n=5)$. (D) Cytotoxicity assays using mitoxantrone to evaluate the effect of silencing ABCG 2 in the 2 I nM single-step clone. Dose-response curves were derived from six independent experiments using the CCK-8 assay for 2 I nM cells with I 2.5 nM siG2-2 siRNA ( $\square$ ) and 2 I nM cells with I2.5 nM siNeg ( $\mathbf{A}$ ). The mean values from six independent experiments are shown with error bars as s.e.m.

acetylated histone (revealed by the ChIP assay) in the ABCG2 proximal promoter is specific to the $A B C G 2$ promoter and specifically due to drug selection. Doxorubicin selection did not alter the levels of global core histone acetylation, which are undetectable. As a control, HDAC inhibition by depsipeptide results in hyperacetylation of $\mathrm{H} 3$ in the whole-cell lysate, again supporting the specificity of the reduced HDAC1 bound to the $A B C G 2$ promoter (Figure $5 C$ ). The expression of HDAC1 was not significantly different among the various sublines.

\section{DISCUSSION}

Doxorubicin is a chemotherapeutic agent principally used for the treatment of solid tumours, especially breast cancer and lymphoma (Minotti et al, 2004). Although doxorubicin can work through various mechanisms, it is still not immune to the MDR phenotype and, consequently, the development of resistance to doxorubicin has been well documented in a broad range of cell lines (Shen et al, 1986; Barrand et al, 1994; Mehta, 1994). To more closely examine the regulation of $\mathrm{ABC}$ transporter expression at concentrations similar to those in vivo, we established several single-step doxorubicin-selected clones with three different cancer cell lines and found that these clones did not express $A B C B 1$ (Figure 1). Although $\mathrm{ABCC} 2$ and $\mathrm{ABCC} 4$ were overexpressed at the mRNA level, ABCG2 was the only transporter responsible for resistance in these clones (Figures 3 and 4). In addition, a podophyllotoxin derivative, etoposide (Hande, 1998), was also able to solicit the same response in MCF-7 cells after single-step selection (Figures $1 \mathrm{~B}$ and $2 \mathrm{C}$ ). Moreover, the selection of IGROV-1 and S-1 cancer cells with 14 or $21 \mathrm{~nm}$ doxorubicin also resulted in the over- expression of ABCG2 (Figure 1C and D). We cannot completely rule out the possibility that a pre-existing cell with high expression of $A B C G 2$ was present in the parental population; nevertheless, we found an overexpression of $A B C G 2$ following low-dose selection in three independent cell lines originating from different tissues using two different drugs. In addition, the lack of enrichment in the putative mammary cancer stem cell surface phenotype, CD44+I CD24- (Al-Hajj et al, 2003) (Supplementary Figure 1), in the single-step doxorubicin-selected MCF-7 clones further supports the theory of adaptation over selection with these studies.

Doxorubicin and etoposide are structurally different but they are both topoisomerase II inhibitors and this may explain the similarities in the response of cells to selection with these agents; however, further studies will be required to establish this. Others have also investigated the effects of single-step doxorubicin selection on the human sarcoma cell line MES-SA and have reported an increase in $A B C B 1$ expression (Chen et al, 1994; Beketic-Oreskovic et al, 1995); yet previous studies were performed before the identification of ABCG2 as a major $A B C$ transporter in the area of MDR. In addition, the authors used $40 \mathrm{~nm}$ doxorubicin for 14 days, nearly two-fold higher than the concentration we employed. We have examined the endogenous levels of $A B C G 2$ in the MES-SA parental cells, and it is not detectable by real-time PCR (data not shown). It is possible that in this cell type, $A B C B 1$ is responsible for resistance; however, as these original studies did not report other $\mathrm{ABC}$ transporters, we cannot address this question. We will determine in the future if a single-step drug regimen results in the same $A B C$ transporters being overexpressed in vivo.

ABCG2 is a half-transporter that appears to have a protective role in a variety of stem cells to maintain progenitor cells in an 


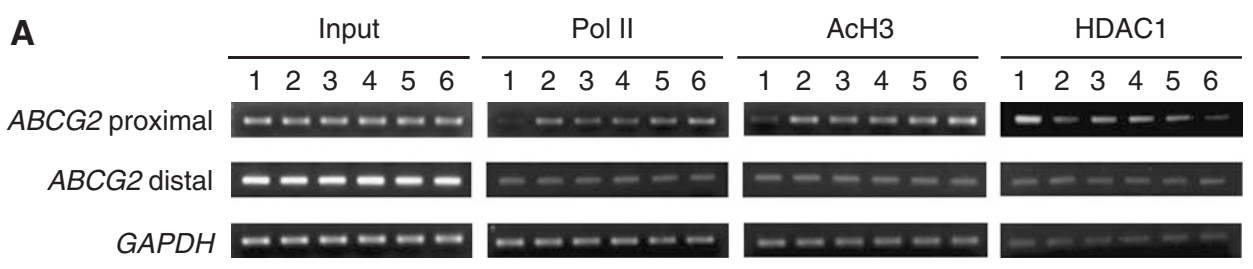

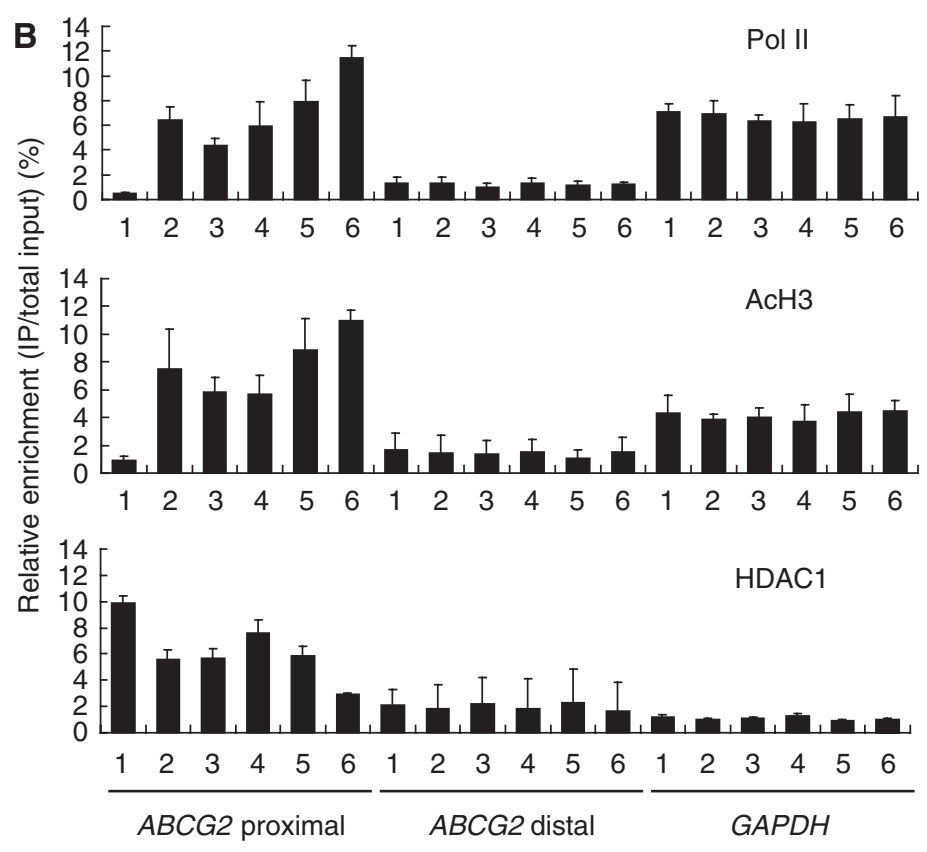

C

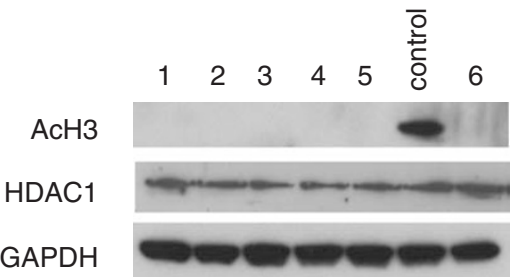

Figure 5 ABCG2 chromatin in the doxorubicin-selected clones is associated with more RNA Pol II and AcH3 but less HDACI than the parental MCF-7 cell line. (A) Chromatin immunoprecipitation assays were performed with parental MCF-7 cells and the doxorubicin-selected clones (Kuo and Allis, I999). A drug-resistant subline MCF7/FLVI 000 (Robey et al, 200 I) was employed as a control. Soluble chromatin used in immunoprecipitations had a typical size of $<0.5 \mathrm{~kb}$ visualisation by gel electrophoresis. RNA Pol II, AcH3 or $\mathrm{HDACl}$ associated with the distal and proximal region in the ABCG2 promoter were analysed by PCR. Lane I, parental MCF-7; lane 2, I 4 nM clone 2; lane 3, I 4 nM clone 6; lane 4, I 4 nM clone I3; lane 5, 2 I nM clone; and lane 6, MCF7/ FLVI000. Input: DNA isolated from the lysate before immunoprecipitation. A representative result from three independent experiments is shown. (B) Quantitative analyses of the occupancy of Pol II, AcH3 or HDACl to the ABCG2 promoter (proximal and distal regions) in the cells. The results are expressed as the percentage of immunoprecipitate (IP) over total input DNA. Error bars show the s.d. of three independent experiments. Lanes I-6 are the same as above. (C) Western blot analysis of $\mathrm{AcH} 3$ and $\mathrm{HDACl}$. Whole-cell lysates were prepared from the parental MCF-7 cells and the various singlestep doxorubicin-selected MCF-7 clones for AcH3 (I7 kDa) and HDACI (65 kDa) detection, respectively. Samples I-6 are as follows: I, parental MCF-7; 2 , $14 \mathrm{nM}$ clone 2; 3, I4 nM clone 6; 4, I4 nM clone 13;5, 2 I nM clone; and 6, MCF7/FLVI 000. As a control, HDAC inhibition by depsipeptide results in hyperacetylation of $\mathrm{H} 3$ in the whole-cell lysate (control lane). Glyceraldehyde-3-phosphate dehydrogenase was used as a loading control for each sample.

undifferentiated state (Zhou et al, 2001) and is often referred to as a stem cell marker. This $\mathrm{ABC}$ transporter is also highly expressed in the placenta, liver and small intestine (reviewed by Krishnamurthy and Schuetz, 2006) where it is influential in the absorption and distribution of xenobiotics. However, to date, the role of this transporter in clinical drug resistance has not been evaluated in any extensive clinical trial (Robey et al, 2007). Interestingly, one smaller study with 59 primary breast cancer patients reported a negative correlation between ABCG2 mRNA expression and response rate and progression-free survival with anthracyclinebased chemotherapy (Burger et al, 2003). Investigators first determined that stem cells from rhesus monkey bone marrow, mouse skeletal muscle and murine embryonic stem cells, which constitute the 'side population' of effluxing cells, expressed ABCG2 (Zhou et al, 2001). In addition, the expression of ABCG2 has been linked to prostate stem cells and mammary gland stem cells (Clayton et al, 2004; Huss et al, 2005). ABCG2 may serve as the primary line of defence against the cytotoxic effects of drug in our single-step-selected clones, as it does in stem cells. However, these cells were not enriched in the putative mammary cancer stem cell surface phenotype, CD44 + /CD24- (Al-Hajj et al, 2003) (Supplementary Figure 1), suggesting that we have not selected for cancer stem cells in these studies and that the epigenetic changes that occurred in the sublines were a result of adaptation as opposed to selection. Others have also reported adaptation of cells to drug pressure as the mechanism of drug resistance (Matsumoto et al, 
1997). Microarray analysis of these single-step doxorubicinselected MCF-7 clones shows that gene expression changes were found primarily in molecular and cellular functions related to cell death, cancer and inflammatory disease (data not shown). This suggests that adaptation to cell stressors such as these may be responsible for turning on the drug resistance genes.

The inhibition of ABCG2 by FTC and the silencing of $A B C G 2$ through siRNA-mediated RNAi showed reversal of drug resistance, confirming that $A B C G 2$ overexpression is responsible for drug resistance (Figure 4). Although ABCC4 expression was seen in these clones, mitoxantrone was not a substrate for ABCC4 (Figure $3 \mathrm{~B}$ ), and ABCC4 did not confer resistance to doxorubicin (Figure 3A). Thus, this transporter is unlikely to be associated with drug resistance in the single-step-selected clones. Using PAGen@UIC (www.uic.edu/pharmacy/depts/pmpcpd/pagen/), we found that there are 11 predicted transcription factors that have binding sites on the promoters of both ABCG2 and ABCC4 (Kamalakaran et al, 2005). Thus, it is possible that the coordinate overexpression of both transporters may be due to an effect of common transcription factors. Further work is necessary to unravel the mechanism of this coordinate overexpression.

In this report, we have shown a reproducible pattern of histone acetylation at the $A B C G 2$ gene that was modified in the clones selected by doxorubicin treatment and in resistant cells (MCF7/ FLV1000) overexpressing ABCG2 (Figure 5). Presumably, HDAC1, specifically bound to the $A B C G 2$ proximal promoter close to the transcriptional initiation site in the parental MCF-7 cells, suppressed histone acetylation at the $A B C G 2$ gene. Therefore, as compared to the parental MCF-7 cells, the weaker association of HDAC1 with the proximal ABCG2 promoter in the selected clones and in MCF7/FLV1000 facilitated histone hyperacetylation and subsequently transcriptional upregulation of $A B C G 2$ (Figure 5). Upregulation of $A B C B 1$, a classical MDR gene, by chemotherapeutic drugs has also been shown to be associated with changes in the spatial and temporal patterns of histone $\mathrm{H} 3$ acetylation (Baker et al, 2005). Further interesting evidence was gleaned from our microarray mRNA expression studies coupled with pathway analysis, indicating that the oestrogen receptor may regulate the expression of $A B C G 2$ (data not shown). This hypothesis is supported by recent work that showed the presence of an oestrogen response element in the ABCG2 gene (Ee et al, 2004). Additional studies are necessary to elucidate the role of the oestrogen receptor in $A B C G 2$ overexpression. To our knowledge, this is the first report of $A B C G 2$ overexpression following singlestep selection with low concentrations of doxorubicin in breast, ovarian and colon cancer cells. Further work will elucidate the triggers that are required to alter expression of these transporters.

\section{ACKNOWLEDGEMENTS}

We thank Dr Michael M Gottesman for his encouragement and helpful discussions. We thank Dr Kapil Mehta (MD Anderson, Houston, TX, USA) for the MCF-7 and MCF7/ADR cell lines and Dr Piet Borst (The Netherlands Cancer Institute) for the HEK294/ 4.63 cells. We thank Sandy Burkett and Gary Stone for performing the spectral karyotyping studies, Dr Toshihiro Suzuki for his assistance with selection of clones and Mr Stephen Wincovitch for his assistance with the confocal studies. The siRNAs used in this study were supplied to NCI by Qiagen Inc. as part of a Collaborative Research Agreement; we thank Eric Lader and Qiagen Inc. for the design and synthesis of the siG2 siRNA. We thank Mr George Leiman for his editorial assistance. This research was supported by the Intramural Research Program of the NIH, National Cancer Institute, Center for Cancer Research. AMC was supported by the NIGMS Pharmacology Research Associate (PRAT) Program.

Supplementary Information accompanies the paper on British Journal of Cancer website (http://www.nature.com/bjc)

\section{REFERENCES}

Al-Hajj M, Wicha MS, Benito-Hernandez A, Morrison SJ, Clarke MF (2003) Prospective identification of tumorigenic breast cancer cells. Proc Natl Acad Sci USA 100: 3983 -3988

Allen JD, Jackson SC, Schinkel AH (2002) A mutation hot spot in the Bcrp1 (Abcg2) multidrug transporter in mouse cell lines selected for doxorubicin resistance. Cancer Res 62: 2294-2299

Allen TM, Cheng WW, Hare JI, Laginha KM (2006) Pharmacokinetics and pharmacodynamics of lipidic nano-particles in cancer. Anti-Cancer Agents Med Chem (Formerly Curr Med Chem Anti-Cancer Agents) 6: $513-523$

Baker EK, Johnstone RW, Zalcberg JR, El-Osta A (2005) Epigenetic changes to the MDR1 locus in response to chemotherapeutic drugs. Oncogene 24: $8061-8075$

Barrand MA, Heppell-Parton AC, Wright KA, Rabbitts PH, Twentyman PR (1994) A 190-kilodalton protein overexpressed in non-P-glycoproteincontaining multidrug-resistant cells and its relationship to the MRP gene. J Natl Cancer Inst 86: 110-117

Beketic-Oreskovic L, Duran GE, Chen G, Dumontet C, Sikic BI (1995) Decreased mutation rate for cellular resistance to doxorubicin and suppression of mdr1 gene activation by the cyclosporin PSC 833. J Natl Cancer Inst 87: $1593-1602$

Burger H, Foekens JA, Look MP, Meijer-van Gelder ME, Klijn JGM, Wiemer EAC, Stoter G, Nooter K (2003) RNA expression of breast cancer resistance protein, lung resistance-related protein, multidrug resistanceassociated proteins 1 and 2, and multidrug resistance gene 1 in breast cancer: correlation with chemotherapeutic response. Clin Cancer Res 9: $827-836$

Calcagno AM, Chewning KJ, Wu CP, Ambudkar SV (2006) Plasma membrane calcium ATPase (PMCA4): a housekeeper for RT - PCR relative quantification of polytopic membrane proteins. BMC Mol Biol 7: 29
Chen G, Jaffrezou JP, Fleming WH, Duran GE, Sikic BI (1994) Prevalence of multidrug resistance related to activation of the mdr1 gene in human sarcoma mutants derived by single-step doxorubicin selection. Cancer Res 54: $4980-4987$

Chen Y, Mickley L, Schwartz A, Acton E, Hwang J, Fojo A (1990) Characterization of adriamycin-resistant human breast cancer cells which display overexpression of a novel resistance-related membrane protein. J Biol Chem 265: 10073-10080

Clayton H, Titley I, Vivanco Md (2004) Growth and differentiation of progenitor/stem cells derived from the human mammary gland. Exp Cell Res 297: $444-460$

Ee PL, Kamalakaran S, Tonetti D, He X, Ross DD, Beck WT (2004) Identification of a novel estrogen response element in the breast cancer resistance protein (ABCG2) gene. Cancer Res 64: 1247-1251

Gottesman M, Fojo T, Bates S (2002) Multidrug resistance in cancer: role of ATP-dependent transporters. Nat Rev Cancer 2: 48-58

Haimeur A, Conseil G, Deeley RG, Cole SP (2004) The MRP-related and BCRP/ABCG2 multidrug resistance proteins: biology, substrate specificity and regulation. Curr Drug Metab 5: 21-53

Hande KR (1998) Etoposide: four decades of development of a topoisomerase II inhibitor. Eur J Cancer 34: 1514-1521

Honjo Y, Hrycyna CA, Yan Q-W, Medina-Perez WY, Robey RW, van de Laar A, Litman T, Dean M, Bates SE (2001) Acquired mutations in the $\mathrm{MXR/BCRP/ABCP}$ gene alter substrate specificity in MXR/BCRP/ABCPoverexpressing cells. Cancer Res 61: 6635-6639

Huss WJ, Gray DR, Greenberg NM, Mohler JL, Smith GJ (2005) Breast cancer resistance protein-mediated efflux of androgen in putative benign and malignant prostate stem cells. Cancer Res 65: 6640-6650

Ishiyama M, Tominaga H, Shiga M, Sasamoto K, Ohkura Y, Ueno K (1996) A combined assay of cell viability and in vitro cytotoxicity with a highly 
Single-step-selected cancer cells overexpress ABCG2

AM Calcagno et al

water-soluble tetrazolium salt, neutral red and crystal violet. Biol Pharm Bull 11: $1518-1520$

Jemal A, Siegel R, Ward E, Murray T, Xu J, Smigal C, Thun MJ (2006) Cancer Statistics, 2006. CA Cancer J Clin 56: 106-130

Kamalakaran S, Radhakrishnan SK, Beck WT (2005) Identification of estrogenresponsive genes using a genome-wide analysis of promoter elements for transcription factor binding sites. J Biol Chem 280: $21491-21497$

Kartner N, Evernden-Porelle D, Bradley G, Ling V (1985) Detection of Pglycoprotein in multidrug-resistant cell lines by monoclonal antibodies. Nature 316: $820-823$

Krishnamurthy P, Schuetz JD (2006) Role of ABCG2/BCRP in biology and medicine. Annu Rev Pharmacol Toxicol 46: $381-410$

Kuo MH, Allis CD (1999) In vivo cross-linking and immunoprecipitation for studying dynamic protein:DNA associations in a chromatin environment. Methods 19: 425-433

Kytola S, Rummukainen J, Nordgren A, Karhu R, Farnebo F, Isola J, Larsson C (2000) Chromosomal alterations in 15 breast cancer cell lines by comparative genomic hybridization and spectral karyotyping. Genes Chromosomes Cancer 28: 308 - 317

Lage H (2003) Drug resistance in breast cancer. Cancer Ther 1: 81-91

Leggas M, Adachi M, Scheffer GL, Sun D, Wielinga P, Du G, Mercer KE, Zhuang Y, Panetta JC, Johnston B, Scheper RJ, Stewart CF, Schuetz JD (2004) Mrp4 confers resistance to topotecan and protects the brain from chemotherapy. Mol Cell Biol 24: 7612-7621

Lorusso V, Manzione L, Silvestris N (2007) Role of liposomal anthracyclines in breast cancer. Ann Oncol 18: vi70-vi73

Maliepaard M, Scheffer GL, Faneyte IF, van Gastelen MA, Pijnenborg ACLM, Schinkel AH, van de Vijver MJ, Scheper RJ, Schellens JHM (2001) Subcellular localization and distribution of the breast cancer resistance protein transporter in normal human tissues. Cancer Res 61: 3458-3464

Matsumoto Y, Takano H, Fojo T (1997) Cellular adaptation to drug exposure: evolution of the drug-resistant phenotype. Cancer Res 57: 5086-5092

Mehta K (1994) High levels of transglutaminase expression in doxorubicinresistant human breast carcinoma cells. Int J Cancer 58: 400 - 406

Minotti G, Menna P, Salvatorelli E, Cairo G, Gianni L (2004) Anthracyclines: molecular advances and pharmacologic developments in antitumor activity and cardiotoxicity. Pharmacol Rev 56: 185-229

Paulusma CC, Bosma PJ, Zaman GJR, Bakker CTM, Otter M, Scheffer GL, Scheper RJ, Borst P, Elferink RPJO (1996) Congenital jaundice in rats with a mutation in a multidrug resistance-associated protein gene. Science 271: $1126-1128$

Perez-Tomas R (2006) Multidrug resistance: retrospect and prospects in anti-cancer drug treatment. Curr Med Chem 13: 1859- 1876
Rabindran SK, He H, Singh M, Brown E, Collins KI, Annable T, Greenberger LM (1998) Reversal of a novel multidrug resistance mechanism in human colon carcinoma cells by fumitremorgin C. Cancer Res 58: $5850-5858$

Rabindran SK, Ross DD, Doyle LA, Yang W, Greenberger LM (2000) Fumitremorgin $\mathrm{C}$ reverses multidrug resistance in cells transfected with the breast cancer resistance protein. Cancer Res 60: 47-50

Reid G, Wielinga P, Zelcer N, De Haas M, Van Deemter L, Wijnholds J, Balzarini J, Borst P (2003) Characterization of the transport of nucleoside analog drugs by the human multidrug resistance proteins MRP4 and MRP5. Mol Pharmacol 63: 1094-1103

Robey RW, Medina-Perez WY, Nishiyama K, Lahusen T, Miyake K, Litman T, Senderowicz AM, Ross DD, Bates SE (2001) Overexpression of the ATP-binding cassette half-transporter, ABCG2 (MXR/BCRP/ABCP1) in flavopiridol-resistant human breast cancer cells. Clin Cancer Res 7: $145-152$

Robey RW, Polgar O, Deeken J, To KW, Bates SE (2007) ABCG2: determining its relevance in clinical drug resistance. Cancer Metast Rev 26: $39-57$

Shen DW, Cardarelli C, Hwang J, Cornwell M, Richert N, Ishii S, Pastan I, Gottesman MM (1986) Multiple drug-resistant human KB carcinoma cells independently selected for high-level resistance to colchicine, adriamycin, or vinblastine show changes in expression of specific proteins. J Biol Chem 261: $7762-7770$

Thigpen JT, Aghajanian CA, Alberts DS, Campos SM, Gordon AN, Markman M, McMeekin DS, Monk BJ, Rose PG (2005) Role of pegylated liposomal doxorubicin in ovarian cancer. Gynecol Oncol 96: 10-18

To KK, Zhan Z, Bates SE (2006) Aberrant promoter methylation of the ABCG2 gene in renal carcinoma. Mol Cell Biol 26: 8572-8585

Wielinga PR, Reid G, Challa EE, van der Heijden I, van Deemter L, de Haas M, Mol C, Kuil AJ, Groeneveld E, Schuetz JD, Brouwer C, De Abreu RA, Wijnholds J, Beijnen JH, Borst P (2002) Thiopurine metabolism and identification of the thiopurine metabolites transported by MRP4 and MRP5 overexpressed in human embryonic kidney cells. Mol Pharmacol 62: 1321 - 1331

Wu CP, Calcagno AM, Hladky SB, Ambudkar SV, Barrand MA (2005) Modulatory effects of plant phenols on human multidrug-resistance proteins 1, 4 and 5 (ABCC1, 4 and 5). FEBS J 272: 4725-4740

Zhou S, Schuetz JD, Bunting KD, Colapietro AM, Sampath J, Morris JJ, Lagutina I, Grosveld GC, Osawa M, Nakauchi H, Sorrentino BP (2001) The ABC transporter Bcrp1/ABCG2 is expressed in a wide variety of stem cells and is a molecular determinant of the side-population phenotype. Nat Med 7: $1028-1034$ 